\title{
Electron acceleration by a wavy shock front: details on angular distribution
}

\author{
Marek Vandas and Marian Karlický \\ Astronomical Institute, Academy of Sciences, 25165 Ondřejov, Czech Republic \\ email: vandas@ig.cas.cz, karlicky@asu.cas.cz
}

\begin{abstract}
We studied numerically electron acceleration by a perpendicular wavy shock. Distribution function of accelerated electrons is highly anisotropic, with many sharp peaks. The peaks are caused by (usually single) reflections of electrons by the shock and subsequent transmission.
\end{abstract}

Keywords. acceleration of particles, shock waves, Sun: corona

\section{Introduction}

Electrons accelerated at a nearly perpendicular shock wave have been observed in 1970s and the process first theoretically treated by Wu (1984) and Leroy \& Mangeney (1984). Vandas (1989) and Krauss-Varban \& Wu (1989) have identified the process as a drift acceleration of electrons inside a shock layer. Zlobec et al. (1993) suggested qualitatively that the acceleration of electrons could be more efficient when a shock front was wavy. In a series of papers, Vandas \& Karlický $(2000,2005,2010)$ examined this possibility quantitatively. They found that accelerated electrons had an unexpectedly anisotropic angular distributions with many sharp peaks. Here we investigate causes of this peculiarity.

\section{Results}

Fig. 1 shows a model of a wavy shock. The shock has a sine-like shock front with the amplitude $A$ and "wavelength" $L$ (amplitude is exaggerated in the figure). Upstream magnetic field is parallel to a smoothed shock front (perpendicular shock wave). To calculate motion of electrons inside the shock layer, the wavy shock is converted into a plane shock wave with $B_{n}$, the normal component of the upstream homogeneous magnetic field to the wavy shock, varying. Electron trajectories are calculated numerically in guiding centre approximation. The shock geometry causes that all upstream electrons are eventually transmitted downstream through the shock after an interaction with it. The model and calculation method are described in detail by Vandas \& Karlický (2000). Parameters of the shock were taken the same as in the cited paper: the upstream magnetic field strength $B_{1}=0.5 \mathrm{mT}(5 \mathrm{G})$, the shock velocity $V_{1}=1000 \mathrm{~km} \mathrm{~s}^{-1}$, the shock thickness $d=50 \mathrm{~m}$, the magnetic field jump at the shock $\nu=1.6$ (corresponding to the Mach number 1.5 and $\beta=0.035), L=1000 \mathrm{~km}, A=100 \mathrm{~km}(A / L=0.1)$. These parameters describe a coronal shock. The initial distribution function of electrons (seed population) is used the same as in Vandas \& Karlický (2000), a kappa function describing halo electrons (suprathermal tail).

Fig. 2a shows an angular distribution of accelerated electrons at a particular place in the downstream region. Their distribution function $f(v, \alpha)$ is plotted relatively to the initial distribution function $f_{i}(v)$ and this value $f / f_{i}$ is displayed as a function of the pitch angle $\alpha$; the angular resolution is $0.5^{\circ}$. The velocity $v$ in the plots corresponds to electron energy of $3 \mathrm{keV}$. Fig. 2a demonstrates a very anisotropic distribution function, 


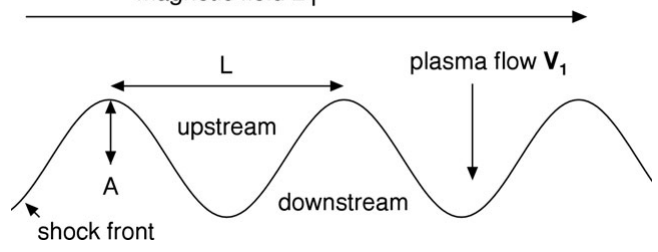

Figure 1. Model shock wave

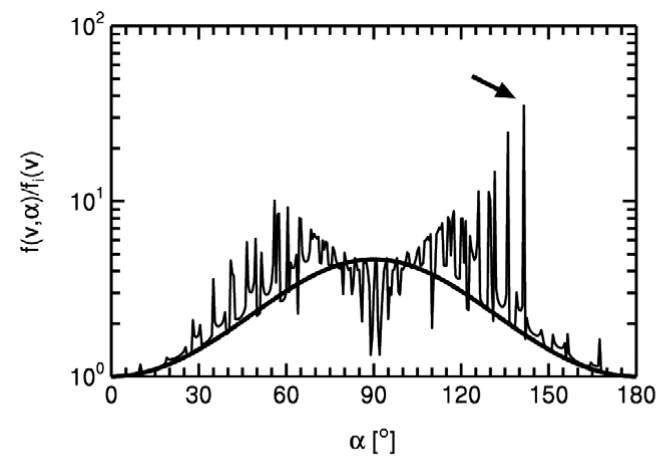

(a)

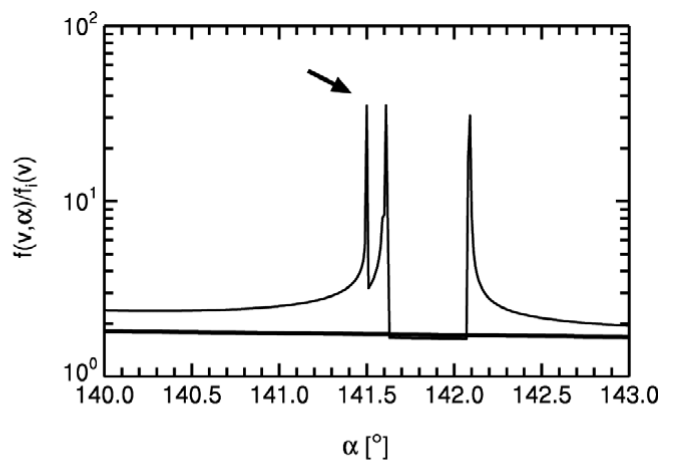

(b)

Figure 2. (a) Angular distribution function $f$ of accelerated downstream electrons for energy $3 \mathrm{keV}$ with dependence on the pitch angle $\alpha$. (b) Detailed view on a selected interval of pitch angles. The angular distribution is plotted relatively to the initial distribution function $f_{i}$ which is isotropic. The smooth thick lines show the angular distribution of accelerated downstream electrons at a plane perpendicular shock.

with many sharp peaks. The highest peak is labeled by an arrow (at $\alpha=141.5^{\circ}$ ) and a part of angular distribution around it is displayed in Fig. $2 \mathrm{~b}$ with the higher angular resolution of $0.01^{\circ}$. The mentioned peak is again labeled by the arrow, and apart of it, additional peaks appear which were not present in Fig. 2a with the lower resolution. It points to a high sensitivity to electron initial values. Due to the wavy form of the shock, electrons may interact with the shock many times. So one can speculate that sharp peaks in Fig. 2, indicating a significantly higher energy gain of electrons, are caused by a sudden increase in a number of interactions for particularly suited combinations of initial parameters. However, a detailed analysis shows that it is not the case. The peaks occur when conditions are suitable for a reflection at the shock. Usually an electron undergoes many transmissions through the shock where the energy gain is small, the decisive role plays a reflection. Fig. 3 demonstrates the situation. Fig. 3a (its right part) shows a trajectory of an electron with the final energy $3 \mathrm{keV}$ and pitch angle $141.50^{\circ}$. It is the case labeled by the arrows in Fig. 2. The electron is one time reflected at the shock (denoted by " $\mathrm{r}_{1}$ ") and eleven times transmitted (denoted by "t" with consecutive numbers in its subscript). Its initial energy was $0.73 \mathrm{keV}$ and pitch angle $93.52^{\circ}$. The trajectories of the electron within the shock are plotted in the left part of the figure and also labeled by "r" and "t". As it has been described, the shock layer is modeled by a plane shock wave. Mutual positions of trajectories do not correspond to reality, they are shifted to approximately match crossings of the shock front in the right part of the figure. Vertical and horizontal directions are not in scale, the latter one is many times zoomed in and differently for the left (much more times) and right parts. Fig. 2b shows a sharp decrease of the labeled peak for a higher $\alpha$. And indeed, only a tiny increase of it changes the situation. Fig. 3b displays an electron with the final energy $3 \mathrm{keV}$ and pitch 


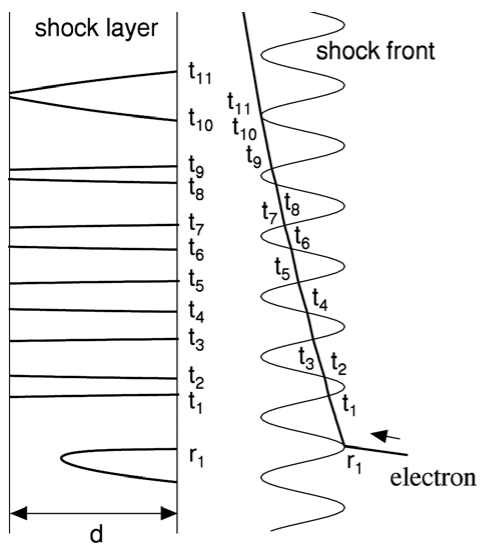

(a)

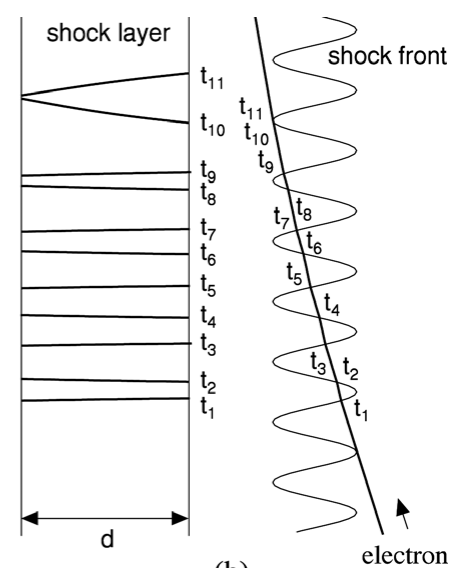

(b)

Figure 3. Trajectories (thick lines) of an electron with the final energy $3 \mathrm{keV}$ and pitch angle (a) $\alpha=141.50^{\circ}$ and (b) $\alpha=141.51^{\circ}$ around and inside the shock. More description is in the text.

angle $141.51^{\circ}$. The electron missed the reflection and it is only eleven times transmitted. Consequently, its energy gain is significantly lower, it had the initial energy $2.13 \mathrm{keV}$ and pitch angle $144.27^{\circ}$. The second peak in Fig. $2 \mathrm{~b}$ is a result of only one reflection and one transmission. Number of interactions plays a secondary role but it can explain decrease of values in Fig. $2 \mathrm{a}$ around $\alpha \approx 90^{\circ}$. Electrons with such pitch angles move slowly along the shock front and it suppresses the number of interactions (units in contrast to tens for other pitch angles).

\section{Conclusions}

Distribution function of accelerated electrons at a wavy shock front is highly anisotropic, with many sharp peaks. The peaks are not caused by many interactions of electrons with the shock front, but by their rare reflections at the shock; for specific values of pitch angles and energies. It follows that a regularly oscillating wave pattern of the shock front (as in our model) is not a necessary condition for these anisotropic distributions. One can expect that similar results would be obtained for an irregular wavy shock.

\section{Acknowledgment}

This research was supported by grants 205/09/0170 from GA ČR, IAA300030805 and IAA300030701 from AV ČR, and by ESA PECS contract 98068.

\section{References}

Krauss-Varban, D. \& Wu, C. S. 1989, J. Geophys. Res., 94, 15367

Leroy, M. \& Mangeney, A. 1984, Ann. Geophys., 2, 449

Vandas, M. 1989, Bull. Astron. Inst. Czech., 40, 189

Vandas, M. \& Karlický, M. 2000, Solar Phys., 197, 85

Vandas, M. \& Karlický, M. 2005, in: B. Fleck, T. H. Zurbuchen \& H. Lacoste (eds.), Proc. Solar Wind 11 - SOHO 16 "Connecting Sun and Heliosphere", ESA SP-592 (Noordwijk: ESA), p. 453

Vandas, M. \& Karlický, M. 2010, A\&AA, submitted

Wu, C. 1984, J. Geophys. Res., 89, 8857

Zlobec, P., Messerotti, M., Karlický, M., \& Urbarz H. 1993, Solar Phys., 144, 373 